Journal of Computer Science 3(10): 786-792, 2007

ISSN 1549-3636

(C) 2007 Science Publications

\title{
GOSTE: Group Oriented Simulation Training Environment
}

\author{
${ }^{1}$ Korichi Ahmed and ${ }^{2}$ Belattar Brahim \\ ${ }^{1}$ Department of Mathematics and Computer Science, \\ University of Ouargla 30000, Algeria \\ ${ }^{2}$ Department of Computer Science, \\ University Hadj Lakhdar of Batna 05000, Algeria
}

\begin{abstract}
The purpose of this study is to explore the contribution of the CSCW (Computer Supported Cooperative Work) in the collaborative project conduct and more specifically in the conduct of a simulation training project. We justify the adequacy of the CSCW concepts with the method of conduct of a simulation project. The practice of the web-based simulation as well as the web-based groupware is treated. This allows us to justify the choice of the BSCW system as support of implementation of a GOSTE on the Web. We illustrate our approach by presenting our experiment with BSCW system to implement such an environment.
\end{abstract}

Key words: Simulation modeling, collaborative simulation, Simulation Training, BSCW, CSCW, WWW

\section{INTRODUCTION}

We observe that an increasing number of enterprises and individuals are equipped with high-tech computers and various accessories (Web cams, scanners, earphones, etc.) Furthermore, democratization of Internet access has led users towards increasingly communitarian and geographically oriented working methods. Users become increasingly demanding and expect computer science tools to assist them in their daily work. One cannot henceforth consider a computer program like a single-station application to be used by an isolated user. The approach that has consisted in the past in investing important sums to develop centralized single-user applications will not be economically profitable in a very near future. Today's tendency favors groupware applications. Many classic single-user applications, such as Text Editors and CAD tools are already available. It is also worth noting to underline that today, worldwide economy has become more interconnected and more competitive, leading to an increasing need for organizations to form joint design teams that collaborate for the life of a project. This is particularly true in the field of modeling and simulation where a simulation project is merely the task of a team rather than that of an isolated person.

In this context the objective of our works is the adaptation of the results of the research originally from the domain of the computer supported cooperative work
(CSCW) by general vocation, to a specific domain, the conduct of a simulation training project.

In the first part of this study we justify the adequacy of the CSCW concepts with the method of conduct of a simulation project. In the second part we present the practices of the web-based simulation and the web-based groupware. In the last part we present our experiment for implementing a web-based simulation groupware with the BSCW system.

\section{SIMULATION AND COOPERATIVE WORKS}

The group dimension of a simulation project: From an external point of view, analyst or engineer is often seen as the unique actor in conducting a simulation project. This is especially true if he or she masters well the simulation software that serves in programming the simulation model. Meanwhile, the practice has shown since a long time that in major simulation projects, a set of collaborators are generally implied (statistician, machine operator, workshop chief, engineer, programmer, client of the study, etc.). Such collaboration can be achieved by internal worker of the enterprise or by external consultants ${ }^{[8]}$, has noted that the demand of consulting in the simulation field has grown faster than the consulting offer since the demand could not be satisfied by local consulting companies or researchers. It is also commonly recognized that to be

Corresponding Author: Korichi Ahmed, ${ }^{1}$ Department of Mathematics and Computer Science, University of Ouargla 30000, Algeria 
able to use correctly and intelligently simulation techniques, it is necessary to have more or less skills in various areas (probabilities and statistics, modeling, programming, etc.). Therefore, due to the diversity of expertise needed, a simulation project must be seen as the responsibility of a group of individuals, acting or not in a structured team where each member plays one or several roles even if the assignment of roles to members is not made in an explicit manner.

Conduct method of cooperative simulation project: Group work can theoretically be organized as per two extreme working methods: subcontracting versus partnership $^{[12]}$. The effective working method often uses a combination of both. In either case, the Project Authority breaks up the work to carry out into parts and delineates it as goals and constraints. He assigns subsets and provides context to each participant or team. Each participant works on his subset according to objectives and constraints imposed, then submits his work-results to the Project Authority who gathers the accomplished subsets, verifies their content, then merges the parts. In the subcontracting method, all constraints must be clearly defined so that each participant may work autonomously. Cooperation is limited to regular interactions and the steps of validation and transmission of information. In the partnership method, interactions between participants take place throughout the process. Interactions make it possible to detect and solve problems as they arise. Theoretically, when a work is done in cooperation with several people, the steps consisting of analysis, design and validation, can be conducted in a sequential way (pure or iterative), or in a concerted way. Of course, the partnership cannot be carried out as a purely sequential method: there would be no possible interactions between the participants.

This overall work-group setting can apply perfectly to the simulation field. Indeed, a project in this domain consists of a set of tasks, each of which requires specific skills. Some of those tasks cannot be accomplished before others are finished. These tasks are accomplished by a work-team placed under the responsibility of a Project Authority and working together on this project in view of obtaining a defined result that is both known and measurable. This workteam, in its size and configuration, varies from a project to another. This variation depends greatly upon the cost and size of the project and is linked to its complexity in both technical and functional aspects; thus, the simulation of a plane requires dozens of specialists and skilled professionals, while the simulation of an electric motor requires few workers in most cases: a programmer and an electro technician. Generally, the team responsible for the realization of the simulation project is composed of: computer specialists (Engineer, Analyst, Programmer, data entry operators, etc.), the main field specialists (Design engineers, Technicians, etc.), the mathematicians (Statistics and Probabilities) and the consulting experts (experts in simulation, experts in professions).

A simulation project can be subdivided into parts and studies of subsets whose complexity is more easily controllable as they have shared relations and constraints. The subsets correspond to possibly different techniques (modeling, statistics and probability, programming, field-trades). This cutting into parts and controllable subsets is essential for its management, its planning and thus to its satisfactory outcome and success. It also enables different scenarios of cooperation between the doers of the project.

The implementation of a simulation project is graded in several stages each of which needs to be followed with the greatest care. It starts with measures taken towards the formalization of the objectives and the terms of references. Next comes the planning (delays, finance, etc.), as well as the acquisition of the computer material and software, the recruitment of highly skilled professionals and the submission of the subsets and objectives entrusted to each one. In the process, the approach is upheld by coordination and follow-up of the teams and work, which can take place through supervision and collaborative work. Figure 1 shows a simple simulation project. A simulation project is usually initiated as result of a need to analyze a real world problem with a view to decision making in a system that cannot be studied directly due to cost or danger. The study usually begins by defining the problem in as much detail as possible. The next step is to form a conceptual model of the physical system in which the problem exists.

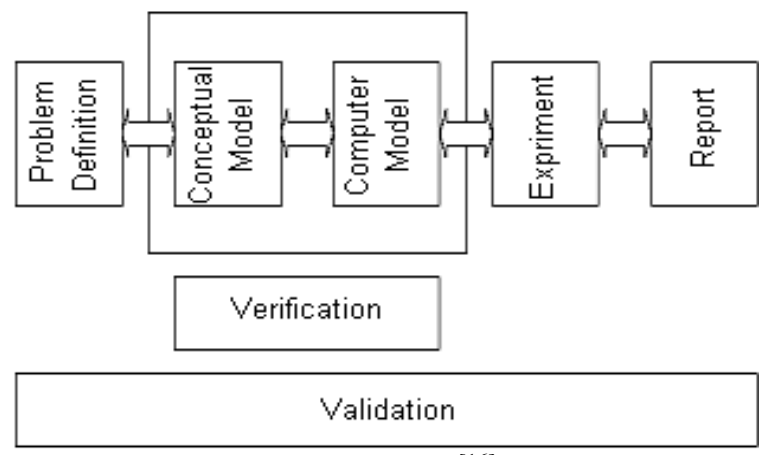

Fig. 1: Simple Simulation Project ${ }^{[16]}$

Diagrammatic techniques are typically used (activity cycle diagrams, event graphs, flow charts, 
block diagrams, etc.). Consideration must also be given to how time advances in the system (time stepped, next event, continuous), to whether or not system activities take deterministic or stochastic time and to how the system changes state (discretely, continuously, or both). Once there is agreement by the involved parties that the conceptual model is an adequate representation of the physical system (validation), it is translated into a computer model, which is then tested (verified) to determine if it conforms to the computer model (although with modern simulation environments the conceptual and computer modeling are often merged). The computer model is then combined with experimental data to attempt to discover more about the problem under investigation. Validation is carried out at all stages of the simulation study to ensure that the various models used do not deviate from the physical system being studied. Statistical analysis of experimental results can either result in recommendations as to how to solve the real world problem, or in further refinement of the problem (as more is discovered about the real world system).

- Cooperation methods: Based on a method for conducting a simulation project as presented hereinabove, we attest that this may be done according to various cooperation methods ${ }^{[17]}$ :

- Asynchronous cooperation: The asynchronous cooperation equates the autonomous working method. In this cooperation method, members work at the same time but in an autonomous way. Each member works individually but it is not excluded that a member use the work of another

- Cooperation in session: Cooperation in session involves presence within the organization. In this co-operation method, members of the group work at the same time, but in an autonomous way. They are available to debate (in Co-temporality) but without sharing data visually

- Cooperation in meeting: The concept of meeting adopted by cooperation in meeting precisely relates to that of a specific project. Clearly identified members work and communicate in Co-temporality while sharing data and discussions. They are assigned roles in connectedness with the aim of the meeting. The organization of their mediation is governed by a policy of speech turn

- Close cooperation: Close cooperation involves a more precise vision of the cooperation. This form of cooperation resembles the concept of cooperative work on the board. Members can work, communicate and interact in real time on all shared project data. Resulting consequences of the group's interventions are directly managed at the level of the data handled

\section{MAJOR TRENDS IN WEB-BASED SIMULATION}

There exist an important amount of works published in simulation conferences dedicated to Webbased simulation topic. In his personal observations ${ }^{[2]}$, discusses how simulation will be affected by Web technologies and focuses on three areas: publication, education and training and simulation programs. Some approaches for using simulation techniques on the Web has also emerged. An example of such approaches consist to transfer a simulation program from a client (Web browser) to a server via HTML forms. The server translate the form's content via CGI scripts into a program that will be executed on the server by a target simulation package (SIMAN, GPSS, QNAP, GPSS, etc.) The simulation results are then transferred via an HTML page back to the client.

Another approach consist to implement a simulation model as a Java applet which can be run on the client side rather than the server side ${ }^{[3]}$. Presents concept and components for a Web-based Simulation Environment. In such an environment, integration of simulation, animation and visualization techniques and tools into the Internet are discussed and problems of the used techniques are shown. In the same context, many other works are dedicated to building simulation models with the Java programming language. Examples of such works are: SIMJAVA ${ }^{[4]}$, SILK ${ }^{[5]}$. On the other hand, works on the integration of CSCW concepts in a modeling and simulation environment (MOSE) are very limited in number and content. The published ones discuss briefly some collaborative aspects of a MOSE without giving serious orientations towards a collaborative MOSE as we have made in $^{[6]}$. Today there exist a large consensus on the practical benefits of such an environment but deeper researches on this topic are still lacking. Meanwhile this didn't prevent that such environments are proposed on the market and the best example to mention is COVISE $^{[7]}$ which supports collaboration between geographically dispersed members and uses virtual reality software and hardware. This environment is however intended to be used on mainframes and use very expensive material and is being experienced by large companies (DaimlerBenz, Aerospace and Airbus). 


\section{J. Computer Sci., 3(10): 786-792, 2007}

\section{WEB-BAED GROUPWARE}

The computer science needs related to any concerted activity are very vast. They require the realization of cooperative software known as groupware witch that take in charge many forms of collaboration and cooperation. Also, although the enormous progress in communication brought by the information technologies and communication via Internet, we notice that all these tools behave in a passive way and they just transmit information. We think that the resorting to Web-based groupware would allow very varied forms of cooperation. This approach is based on the taking into account of the possibilities offered by current technologies in order to make possible the work in groups which is dominant in any current activity using the Internet network. The choice of the Web as platform is justified by the fact that the majority of the groupware which were elaborated in research laboratories are sensitive to the work environment. Installing a groupware outside the place on which was designed is a complex task: You must be able to take into account the diversity of the machines, of the operating systems and the various types of networks. Today the companies need cooperative systems to connect the teams, doesn't matter they are in the opposite building or they are anywhere over the world. Thus the WWW proposes a completely homogeneous layer making it possible to connect all the types of platforms.

A Web-based groupware is software that allows people visiting a site to collaborate via the Web on the creation of documents or the exchange of ideas ${ }^{[10]}$. Experts divide groupware into two main categories: synchronous and asynchronous. Synchronous groupware allows interactivity between users who are all connected at the same time, such as live chats or videoconferences. Asynchronous groupware allows people to share information by leaving messages on a site for others to read when they log in (e.g., E-mail). Web-based groupware can use videoconferencing, Whiteboards, live chat, etc. Each of these features has the ability to turn a Web site into a real platform of collaboration. It is also important to note that today with features like Dynamic HTML and JavaScript, the Web has moved from a simple platform facilitating the sharing of HyperText documents to a real programming platform suited for prototyping and supporting many kind of applications.

A simulation groupware can then be viewed as a Web-based groupware aiming at providing the simulation project team with an efficient platform of collaboration where team members actively work, share documents and ideas about their projects, leading to better results both in term of costs and quality. The main features that a simulation groupware must provide are: a whiteboard and a chat capabilities. A Whiteboard will serve as a shared space in which a document pertaining to the simulation project (simulation model or sub-model, simulation outputs, project documentation, etc.) can be viewed by two or more group members simultaneously. While members view the document, they can make notes or changes using the drawing and/or the annotations capabilities provided by the Whiteboard. A chat capability will serve to engage an on-line exchange of text-based messages between two or more group members. Such a discussion can be engaged while viewing something on the whiteboard leading to an effective collaboration. This will give to a member the ability to comment or review a work done by another member, find a consensus on some part of the project, get a help on some topic, etc.

\section{IMPLEMENTATION}

There is more than one approach to implement a groupware. One approach consists of using techniques and tools far less refined than those on the market. In this instance, we are confronted with the classic dilemma which consists of working with rudimentary and archaic tools. Another approach consists of using standard Computer Supported Cooperative Work tools that are more or less high-tech. The second approach seems to be simpler and easier to realize on the condition to find a standard and adequate tool for $\mathrm{CSCW}$. Otherwise, we would be obligated to start the work from scratch.

A state of the art groupware capable to support the cooperative work of a team on simulation projects, allowed us to examine the latest and most crucial offers in the domain of the CSCW (MOCCA, COLA, YARN, CSpray, AlephWeb, Tveiws, BSCW, Manicoral, Patern-Ext, DARE and PINAS). Our choice of experimentation groupware was based on BSCW system (Basic Support Cooperative Work). This choice is justified by uncertainty about the available IT infrastructures of all project participants, as well as for its usability and the universality of its access. This solution exempts members from having to install specific customer software on the machines they want to use. It also enables them to work from different places.

The BSCW Shared Workspace System is an Web based groupware system. It is an extension of a standard Web server through the server CGI Application Programming Interface. The central 
metaphor of the system is the shared workspace. A BSCW server (Web server with the BSCW extension) manages a number of shared workspaces, i.e., repositories for shared information, accessible to members of a group using a simple user name and password scheme. Shared workspaces can contain various kinds of information such as documents, pictures, URL links to other Web pages, threaded discussions, member contact information and more. The contents of each workspace are represented as information objects arranged in a folder hierarchy. Of course, in addition to the normal download of information from a Web site, users can also upload information from their local file system into a BSCW workspace. The following are the main features of the system $^{[13]}$ :

- Authentication: People have to identify themselves by name and password before they have access to BSCW workspaces.

- Discussion forums: Users may start a discussion on any topic they like and the system presents the threads in a user friendly manner.

- Access rights: The system contains a sophisticated access rights model which allows, for example, that some users may have complete control over an object in a workspace whereas others have only read access or no access at all.

- Search facilities: Users can specify queries to find objects within BSCW workspaces based on names, content or specific properties such as document author or document modification date. Furthermore, queries may be submitted to Web search engines and the result of the query can be imported into workspaces.

- Document format conversion: These facilities allow users to transform a document into their format of choice, e.g., a proprietary document format into HTML, before downloading it.

- Version management: Documents within a workspace can be put under version control which is particularly useful for joint document production.

- Multi-language support: The interface of the system can be tailored to a particular language by straightforward extensions. Several languages (e.g., French, Spanish, Italian, Russian, Greek and Catalan) have been created by users of the system and are publicly available.

- Event services: A cooperative system has to provide awareness information to allow users to coordinate their work. The event services of the
BSCW system provide users with information on the activities of other users, with respect to the objects within a shared workspace. Events are triggered whenever a user performs an action in a workspace, such as uploading a new document, downloading an existing document, renaming a document and so on. The system records the events and presents the recent events to each user. In addition, users can request immediate email messages whenever an event occurs and so-called daily activity reports which are sent to them daily and informs them about the events within the last $24 \mathrm{~h}$.

The working philosophy of the BSCW system and these functionalities enabled us to implement perfectly the concepts and the various methods of possible cooperation of a simulation project. The implementation of the cooperation in meeting and the close cooperation is done only by the possibility of the use of the suitable external software (ex. MSNetMeeting.). Figure 2 represents a participant's space who discusses the possibility of the use of the ARENA simulator in the current project with another member via NetMeeting Software.

The experimentation was conducted within the project framework of the end of studies to obtain a diploma in industrial computer science engineering at the University of first Author. The title of the theme was Construction of a simulation model for the performance evaluation of a production system: Case of the national company of industrial gases ENGI. Two students, following their supervisor with the collaboration of two other engineers and a specialist in probabilities and statistics, participated in the experiment. The experimentation project stretched out over six months.

The electronic outset of the project under the BSCW system was the subject of a reservation of a logical space managed by the project chief and of an analogous physical space. The project participants subscribe to this logical space. The logical space is organized as a tree structure of folders and files; chosen because it is a structure familiar to computer science users. In our implementation this folders structure has been used to structure logical space (ex: common space, team spaces, actors private spaces) and the creation of the project artifacts. Rather than assuming that all features of BSCW and NetMeeting were useful, a short survey was conducted to elicit opinion of participants on the interpreted adequacy of this implementation. 


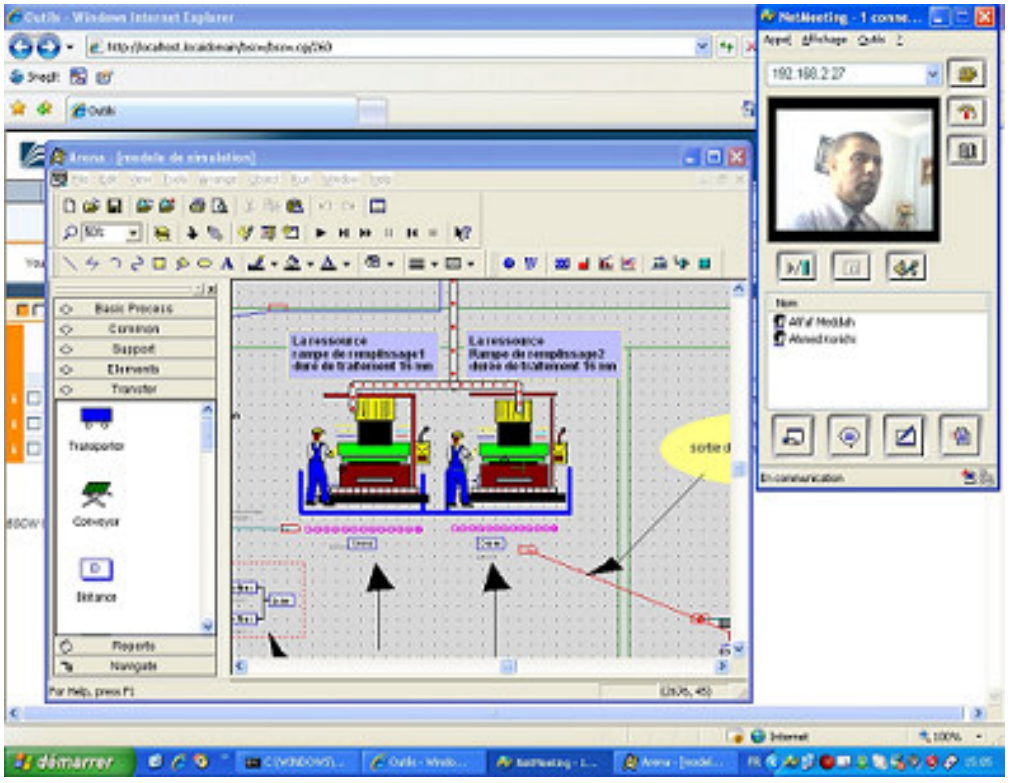

Fig. 2: A snapshot from the implementation: BSCW, NetMeeting and Arena Software in use

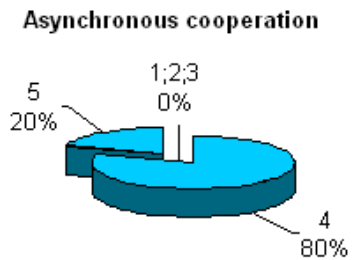

Cooperation in meeting

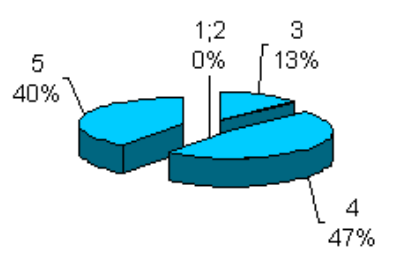

Cooperation in session

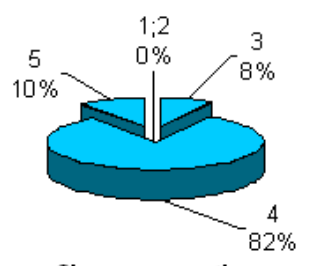

Close cooperation

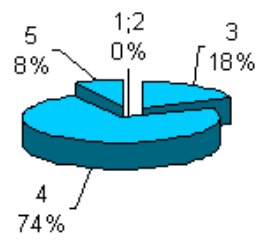

Fig. 3: Results of the participants' evaluation

This led us to take a series of demonstrations and then follow up with a questionnaire. Figure 3 shows the results of the evaluation. Each participant was asked to rate the groupware adequacy on a scale from 1 to 5 for each cooperation method. Participants were also asked to weight their expertise of simulation and computer technology. Hindsight suggests that the 1 to 5 scale should have been broader (to present a wider range of options) and that expertise should have been evaluated against other factors rather than just a number. Although the cross-section of the simulation community was small, the result of this experiment is extremely clear.

\section{CONCLUSION}

In this study, a web-based simulation groupware is considered. We have examined different facets of cooperative work in the context of a simulation project. An example of implementation was presented and results from a short survey were discussed. The ideas proposed are not tied to a specific simulation tool and can be used with any tool. A web-based simulation groupware can also be used as a cooperative learning support to teach simulation techniques. In this case, the teacher can act as the project manager and students act as team members.

\section{REFRENCES}

1. Mackulak, G.T., J.K. Cochran and P.A. Savory, 1996. Ascertaining Important Features for Industrial Simulation Environments. Simulation, 63: 211-211.

2. Fishwick, P.A., 1996. Web-based Simulation: Some Personal Observations, in Winter Simulation Conference, San Diego, CA, pp: 772-779.

3. Seibt, F., M. Schumann and J. Beikirch, 1998. Concept and Components for a Web-based Simulation Environment (WBSE. International Conference on Web-Based Modeling and Simulation, San Diego CA, pp: 189-194. 
4. Fred Howell and Ross McNab, 1998. SIMJAVA: A Discrete Event Simulation Package for Java with Applications in Computer Systems Modeling. First International Conference on Web-based Modelling and Simulation, San Diego CA. Society for Computer Simulation, pp: 51-56.

5. Kilgore, R. and K. Healy Java, 1997. Enterprise Simulation and the Silk Simulation Language. In Proceedings of the 1998 SCS International Conference on Web-Based Modeling and Simulation, pp: 195-200.

6. Belattar, B., A. Zidani and M. Djoudi, 1997. Vers Un Environnement de Simulation Cooperative, MOSIM'97 Conference, pp: 483-499.

7. Ulrich Lang, 1994. COVISE: Collaborative Visualization and Simulation Environment, RUS: Regionales Rechenzentrum Universität Stuttgart. http://www.unistuttgart.de/RUSuser/vis/Covise/covise.html.

8. XJerry Banks, B. Barry, Burnette, Henry Kowloski and James D. Rose, 1994. Introduction to SIMAN $\mathrm{V}$ and CINEMA V. John Wiley and Sons, Inc.

9. Gronbaek, K., Jens A. Hem and Ole L. Madsen Lennert Sloth, 1994. Systems: A Dexter-Based Architecture. Communication of the ACM, 37: 65-74.
10. Tony Jaros, 2005. The Meeting of the (Virtual) Minds, <http://www.internet.com/>

11. Appelt, W., 1999. WWW Based Collaboration with the BSCW System, Proc. of SOFSEM'99, pp: 66-78.

12. Tarpin-Bernard, F., 1997. Travail Coopératif Synchrone Assisté par Ordinateur Approche AMFC, Ph.D Theses, University of Lyon.

13. BSCW, 2007. The BSCW Website, <http://bscw.gmd.de>

14. Bentley, Richard, Busbach, Uwe and Sikkel Klaas, 1996. The Architecture of the BSCW Shared Workspace System. In Proc. 5th ERCIM/W4G Workshop "CSCW and the Web", Arbeitspapiere der GMD 984, GMD, Sankt Augustin, pp: 31-42.

15. Bentley, R., T. Horstman and J. Trevor, 1997. The World Wide Web as Enabling Technology for CSCW: The Case of BSCW, JCSCW, 6: 111-134.

16. Taylor, S.J.E., 2000. NetMeeting: A Tool for Collaborative Simulation Modeling. I. J. of Simulation Systems, Sci. and Tech., 1: 59-68.

17. Korichi, A. and B. Belattar, 2006. Model of a Cooperative Simulation Project. Asian J. Inform. Tech., (AJIT), 5: 955-962. 